OPEN ACCESS

Edited by:

Lingqian Wu,

Central South University, China

Reviewed by:

Yong Feng,

University of South China Affiliated Changsha Central Hospital, China

Yilai Shu,

Fudan University, China

${ }^{*}$ Correspondence:

Tao Yang

yangtfxl@sina.com

Hao Wu

wuhao622@sina.cn

${ }^{t}$ These authors have contributed equally to this work and share first authorship

Specialty section:

This article was submitted to Genetics of Common and Rare

Diseases,

a section of the journal

Frontiers in Genetics

Received: 16 September 2021 Accepted: 16 November 2021 Published: 08 December 2021

Citation:

Chen P, Wang L, Chai Y, Wu H and Yang $T$ (2021) Detection and Functional Verification of Noncanonical Splice Site Mutations in Hereditary Deafness.

Front. Genet. 12:773922. doi: $10.3389 /$ fgene.2021.773922

\section{Detection and Functional Verification of Noncanonical Splice Site Mutations in Hereditary Deafness}

\author{
Penghui Chen ${ }^{1,2,3,4 t}$, Longhao Wang ${ }^{1,2,3 t}$, Yongchuan Chai ${ }^{1,2,3 t}$, Hao $\mathrm{Wu}^{1,2,3 *}$ and \\ Tao Yang ${ }^{1,2,3 *}$
}

${ }^{1}$ Department of Otolaryngology - Head and Neck Surgery, Shanghai Ninth People's Hospital, Shanghai Jiaotong University School of Medicine, Shanghai, China, 'Ear Institute, Shanghai Jiaotong University School of Medicine, Shanghai, China, ${ }^{3}$ Shanghai Key Laboratory of Translational Medicine on Ear and Nose Diseases, Shanghai, China, ${ }^{4}$ Department of Otorhinolaryngology - Head and Neck Surgery, Xinhua Hospital, Shanghai Jiaotong University School of Medicine, Shanghai, China

Splice site mutations contribute to a significant portion of the genetic causes for mendelian disorders including deafness. By next-generation sequencing of 4 multiplex, autosomal dominant families and 2 simplex, autosomal recessive families with hereditary deafness, we identified a variety of candidate pathogenic variants in noncanonical splice sites of known deafness genes, which include c.1616+3A > T and c.580G > A in EYA4, c.32257_322-8del in PAX3, c.991-15_991-13del in DFNA5, c.6087-3T > G in PTPRQ and c. $164+5 G>A$ in USH1G. All six variants were predicted to affect the RNA splicing by at least one of the computational tools Human Splicing Finder, NNSPLICE and NetGene2. Phenotypic segregation of the variants was confirmed in all families and is consistent with previously reported genotype-phenotype correlations of the corresponding genes. Minigene analysis showed that those splicing site variants likely have various negative impact including exon-skipping (c.1616+3A > T and c.580G > A in EYA4, c.991-15_99113del in DFNA5), intron retention (c.322-57_322-8del in PAX3), exon skipping and intron retention (c.6087-3T > G in PTPRQ) and shortening of exon (c.164+5G > A in USH1G). Our study showed that the cryptic, noncanonical splice site mutations may play an important role in the molecular etiology of hereditary deafness, whose diagnosis can be facilitated by modified filtering criteria for the next-generation sequencing data, functional verification, as well as segregation, bioinformatics, and genotype-phenotype correlation analysis.

Keywords: splice site mutation, RNA splicing, minigene, hereditary deafness, next-generation sequencing

\section{INTRODUCTION}

RNA splicing refers to the process of removing introns from the initial transcript (preRNA), transcribed from its DNA template, and connecting exons to form a continuous RNA molecule. In eukaryotic cells, sequences near the splicing sites of preRNA are conserved, which include the GT bases at the $5^{\prime}$ donor site of the intron, the AG bases at the $3^{\prime}$ acceptor site, the branch point composed of the polypyrimidine trace and splicing regulatory sequences such as exonic splicing enhancer (ESE), exonic splicing silencer (ESS), intronic splicing enhancer (ISE) and intronic splicing silencer (ISS) (Glisovic et al., 2008; Anna and Monika, 2018). The existence of these conserved sequences ensures the accurate RNA splicing, while mutations in these sequences may lead to structural alteration of the protein products and a variety of genetic disorders (Wang et al., 2012). 
According to the human gene mutation database (HGMD), approximately $9 \%$ of the pathogenic mutations are splice site mutations (http://www.hgmd.cf.ac.uk/ac/index.php). The splice site mutations can be divided into four categories based on their locations: (I) canonical splice site (CSS) mutations at the intronic +1 and +2 positions of the $5^{\prime}$ donor splicing site and the -2 and -1 positions of the $3^{\prime}$ receptor splicing site; (II) mutations in the junction regions from exonic $3 \mathrm{bp}$ to intronic $6 \mathrm{bp}$ and from intronic $12 \mathrm{bp}$ to exonic $2 \mathrm{bp}$, excluding the classic region; (III) exonic missense or synonymous mutations in the ESE, ESS and UTR region; (IV) mutations in deep intron such as the branch point, ISE and ISS (Cartegni et al., 2002). The type II, III, and IV mutations were referred to as noncanonical splice site (NCSS) mutations. Like CSS mutations, NCSS mutations can abolish an existing donor or receptor splicing site, often resulting in loss of a whole exon (exon skipping). It can also indirectly activate a hidden splice site in exons or introns, sometimes resulting partial intron retention or exon shortening (Booth et al., 2018). In some cases, intronic or exonic mutations may create a new splice site, resulting in partial intron retention, exon shortening or formation of pseudo exons (VazDrago et al., 2017).

Detection of NCSS mutations can be difficult, because the targeted sequences are not rigidly conserved as the canonical ones and the locations of mutations are far more variable (Anna and Monika, 2018). To this end, bioinformatic analyzing tools, such as Human Splicing Finder (HSF), Splice Site Prediction by Neural Network (NNSPLICE) and NetGene2, have been developed to evaluate the possible pathogenic effect of the splice site mutations (Anna and Monika, 2018). The results of the computational analysis, however, are only predictive. For NCSS mutations, functional studies such as in vivo RNA sequencing and in vitro minigene analysis, are often needed to verify their exact effect on RNA splicing (Anna and Monika, 2018).

Hereditary deafness has been known for its tremendous genetic heterogeneity (Kremer, 2019; Sheffield and Smith, 2019). Facilitated by recent development and widespread implication of nextgeneration sequencing (NGS), vast amounts of pathogenic variants in over 100 deafness-causative genes have been documented in recent years (https://hereditaryhearingloss.org/). Variants at NCSS, however, are often classified as variants of unknown significance (VUS) due to limited research methods to readily distinguish the pathogenic ones from the large number of benign polymorphisms (Wang et al., 2012). To this day, NCSS mutations have been reported and functionally verified in only a handful of deafness-causative genes, including a c.1282-12T $>\mathrm{A}$ in EYA4 lead to non-syndromic deafness DFNA10 (Hildebrand et al., 2007), a c.6050-15G > A mutation in CDH23 lead to atypical USH1 syndrome (Valero et al., 2019), and a number of NCSS mutations involving splicing of exon 8 in DFNA5 (Booth et al., 2018).

In this study, we identified 6 NCSS and 1 CSS mutations in EYA4, PAX3, DFNA5, PTPRQ and USH1G from 4 multiplex, autosomal dominant families and 2 simplex, autosomal recessive families with hereditary deafness. A workflow has been proposed for analyzing the NCSS variants in genetic hearing loss, including NGS with modified filtering criteria for NCSS mutation, familial segregation and genotype-phenotype correlation analysis, bioinformatic prediction and functional verification.

\section{MATERIALS AND METHODS}

\section{Subjects}

Probands and participating members of the 4 multiplex, autosomal dominant families and 2 simplex, autosomal recessive families were recruited through the Department of Otolaryngology-Head and neck surgery, Ninth People's Hospital, Shanghai Jiaotong University School of medicine, Shanghai, China. The pedigrees are shown in Figure 1. All subjects gave signed, informed consent to participate this study, which was approved by the ethics committee of Ninth People's Hospital, Shanghai Jiaotong University School of Medicine.

\section{Next-Generation Sequencing}

Genomic DNA samples were extracted from venous blood. The exons and flanking introns of 144 deafness-causative genes (Supplementary Table S1) were captured using the MyGenotics gene enrichment system (MyGenotics, Boston, MD, United States) and sequenced using the Illumina HiSeq 2000 sequencer (Illumina, San Diego, CA, United States) as previously described (Chen et al., 2016). The human genome GRCh37/hg19 was used for sequence alignment. Conventional filtering criteria were applied in the initial round of sequencing data analysis, in which only nonsynonymous variants in the coding region and the CSS variants were interrogated. In the second round of analysis, candidate variants were expanded to all intronic variants within 50 nucleotide bases from the exon boundary and synonymous/nonsynonymous variants in the exons. The minor allele frequency (MAF) for the candidate variants was set as 0.005 or less for recessive inheritance and 0.0005 or less for dominant inheritance (Shearer et al., 2014), based on public databases ESP (http://evs.gs.washington.edu/EVS/), GnomAD (https:// gnomad.broadinstitute.org/) and an in-house Chinese Han population database. The pathogenicity of candidate variants was predicted by computational tools MutationTaster, PROVEAN, SIFT and Polyphen-2. Intrafamilial segregation of the candidate variants were confirmed by Sanger sequencing of all participating family members.

\section{Bioinformatics Analysis}

Three bioinformatic analyzing tools for splicing site variants were used in this study, including HSF version 3.1 (http://www.umd. be/HSF3/HSF.shtml), NNSPLICE version 0.9 (https://www. fruitfly.org/seq_tools/splice.html) and NetGene2 (http://www. cbs.dtu.dk/services/NetGene2/).

\section{Minigene Analysis}

The in vitro minigene analysis was performed as previously described (Booth et al., 2018). Wild-type and mutant minigene inserts were directly synthesized (Sangon Biotech, Shanghai, China, Supplementary Table S2) or amplified from the patients' genomic DNA (Supplementary Table S3). The inserts were cloned into the pre-constructed exon-trap vectors pET01 (MoBiTec, Goettingen, Germany), pEGFP-C1 or pcMINI (Wuhan bioegle Biological Technology and Science Co., Ltd., Wuhan, China), all with intrinsic $3^{\prime}$ and $5^{\prime}$ exons separated by a multiple cloning site (MCS). The minigene constructs were then transfected into COS7 cells (ATCC_CRL1651)using LipofectamineTM3000 Transfection 


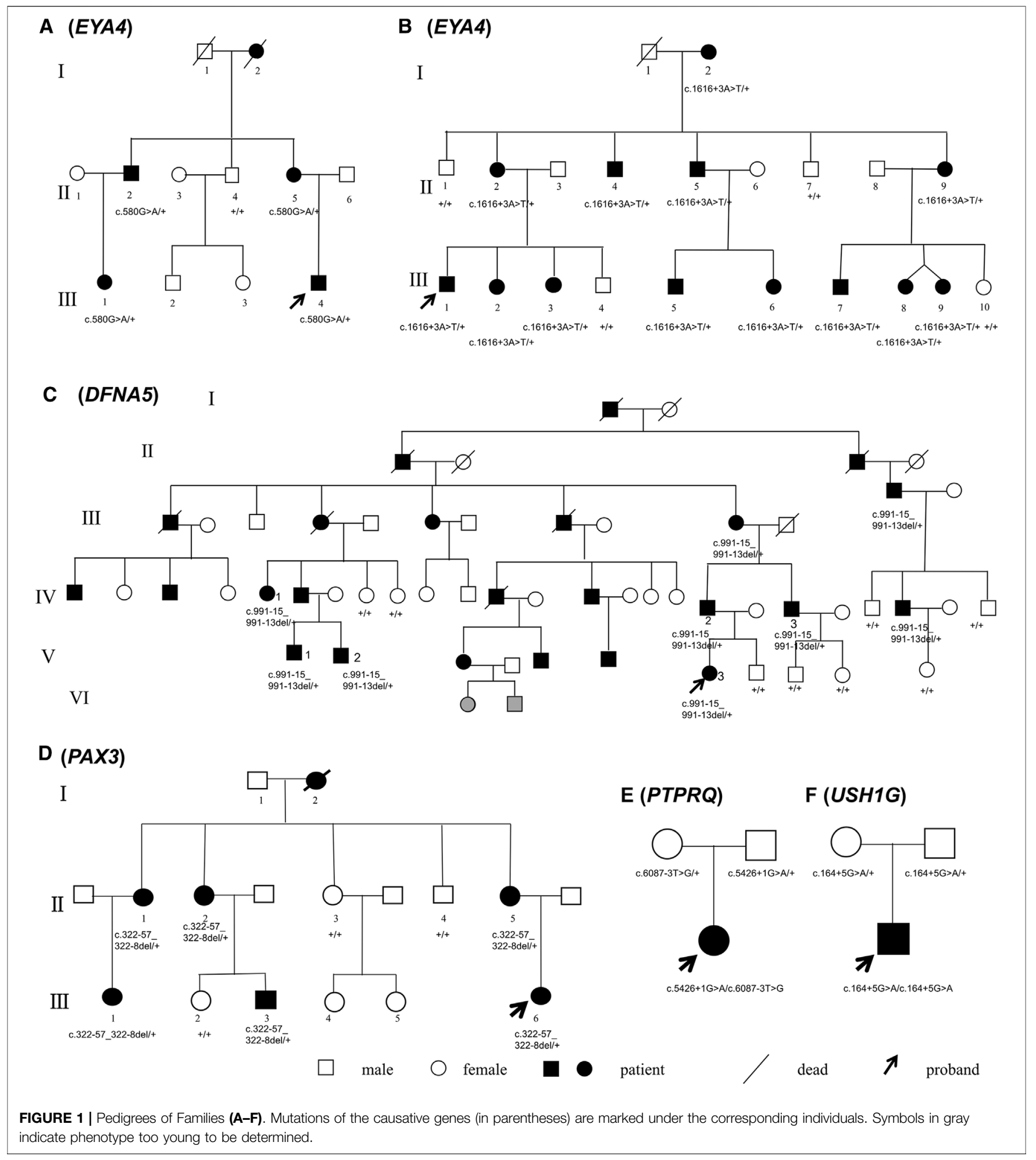

Reagent (Thermo Fisher Scientific, Waltham, Massachusetts, United States). Cells were harvested $36 \mathrm{~h}$ after transfection. The total RNA was extracted using the Trizol method. cDNA was reversely transcribed by TaqMan Reverse Transcription Reagents
(Takara Bio Inc., Japan). The spliced products were PCR amplified with intrinsic primers from the pET01, pEGFP-C1 or pcMINI vectors, detected by agarose gel electrophoresis and Sanger sequencing. 


\section{A (EYA4)}

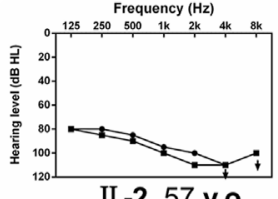

II $-2,57$ y.o.

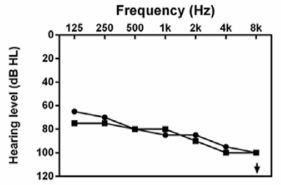

III-1, 38 y.o.

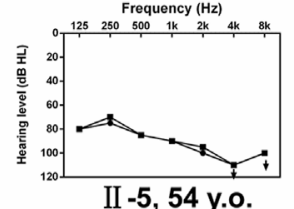

II $-5,54$ y.o.

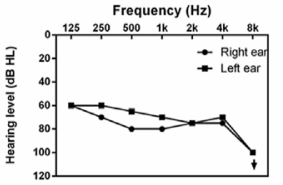

III-4, 31 y.o.
B (EYA4)

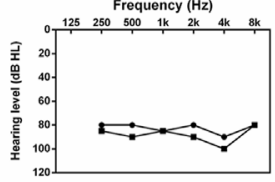

II -2, 70 y.o.

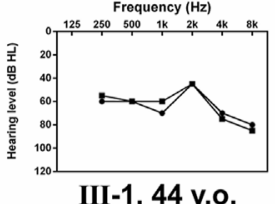

III-1, 44 y.o.

Frequency $(\mathrm{Hz})$

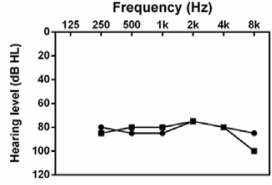

III-6, 47 y.o.

D (PAX3)

C (DFNA5)
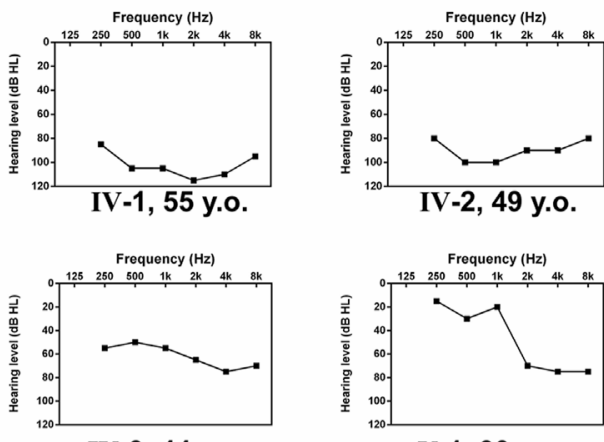

IV -3, 44 y.o.

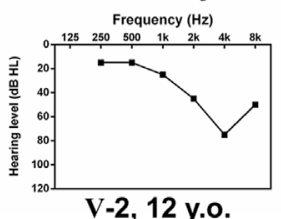

V-2, 12 y.o.

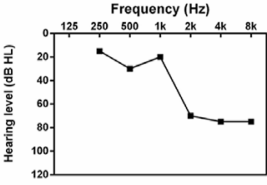

V-1, 30 y.o.

Frequency ( $\mathrm{Hz})$

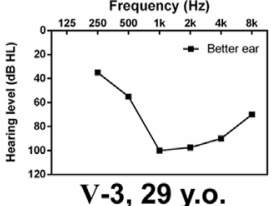

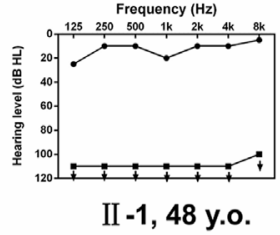

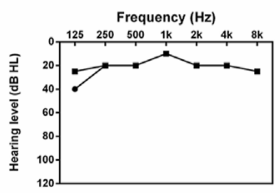

II-5, 32 y.o.

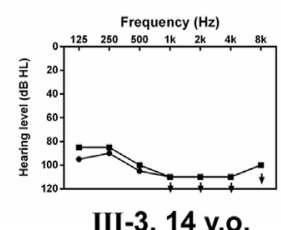

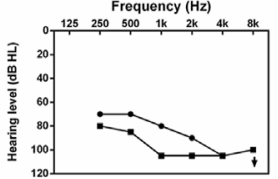

II -5, 60 y.o.

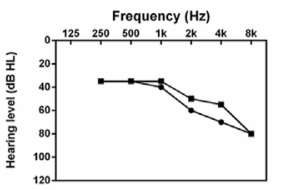

III-2, 48 y.o.

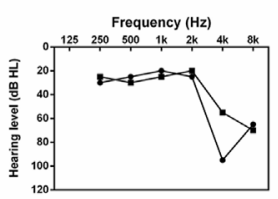

III-7, 31 y.o.

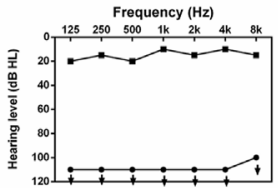

II -2, 45 y.o.

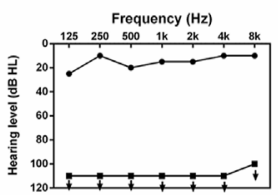

III-1, 27 y.o.

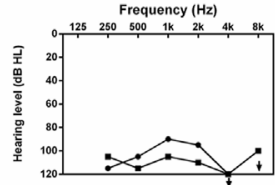

II -9, 54 y.o.

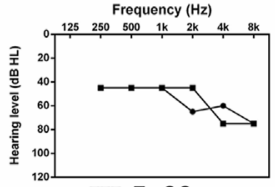

III-5, 39 y.o.

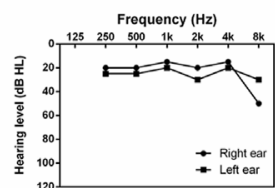

III-8, 26 y.o.

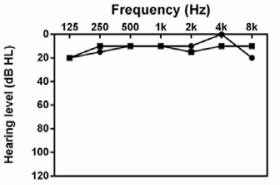

$\because \begin{aligned} & \text { Rightear } \\ & \text { Left ear }\end{aligned}$

FIGURE 2 | Audiograms of the affected individuals in Families (A-D), which shows hearing of both ears (A,B,D) or the better ear (C)

\section{RESULTS}

\section{Clinical Characteristics of the Families With Deafness}

Families A-D exhibited typical autosomal dominant inheritance with multiple affected members spanning at least three generations (Figure 1). For Families A, B and C, audiograms of the affected members were consistent within each family, all showing bilateral, symmetric, progressive sensorineural hearing loss with delayed onsets (Figure 2). No other symptoms were reported. The affected members in Family D show characteristic features of Waardenburg syndrome type III (WS3) including sensorineural deafness, heterochromic iridis, premature graying of the hair, dystopia canthorum, patchy de-pigmentation of the skin, dystrophia canthorum and upper limb anomalies with intrafamilial variation (Table 1). Interestingly, four affected members in Family D exhibited unilateral hearing loss, which is extremely rare in genetic hearing loss. In the two simplex, autosomal recessive families (Figures 1E,F), both affected children had non-syndromic, congenital, profound deafness.

\section{Identification and Verification of the Candidate Pathogenic Variants}

By targeted NGS, we identified candidate pathogenic variants in each of the multiplex, autosomal dominant families, 
TABLE 1 | Characteristic features of WS3 in Family D.

\begin{tabular}{|c|c|c|c|c|c|c|c|}
\hline Status & Member & Deafness & Heterochromic iridis & $\begin{array}{c}\text { Premature graying of the } \\
\text { hair }\end{array}$ & Dystopia canthorum & $\begin{array}{c}\text { Patchy de-pigmentation of } \\
\text { the skin }\end{array}$ & Limb anomaly \\
\hline Affected & $\|-1$ & Left ear & $\mathrm{N}$ & $\mathrm{N}$ & $Y$ & $N$ & $\mathrm{~N}$ \\
\hline Affected & $\|-2$ & Right ear & Both & $Y$ & $Y$ & $\mathrm{~N}$ & $\mathrm{~N}$ \\
\hline Unaffected & II-3 & $\mathrm{N}$ & $\mathrm{N}$ & $\mathrm{N}$ & $\mathrm{N}$ & $\mathrm{N}$ & $\mathrm{N}$ \\
\hline Unaffected & $\|-4$ & $\mathrm{~N}$ & $\mathrm{~N}$ & $\mathrm{~N}$ & $\mathrm{~N}$ & $\mathrm{~N}$ & $\mathrm{~N}$ \\
\hline Affected & $\|-5$ & $\mathrm{~N}$ & $\mathrm{~N}$ & $\mathrm{~N}$ & $Y$ & $N$ & $\mathrm{~N}$ \\
\hline Affected & || $\mid-1$ & Left ear & Right eye & Y & Y & $\mathrm{N}$ & $\mathrm{N}$ \\
\hline Unaffected & III-2 & $\mathrm{N}$ & $\mathrm{N}$ & $\mathrm{N}$ & $\mathrm{N}$ & $\mathrm{N}$ & $\mathrm{N}$ \\
\hline Affected & III-3 & Both & Right eye & $\mathrm{N}$ & Y & $\mathrm{N}$ & $\mathrm{N}$ \\
\hline Unaffected & || $\mid-4$ & $\mathrm{~N}$ & $\mathrm{~N}$ & $\mathrm{~N}$ & $\mathrm{~N}$ & $\mathrm{~N}$ & $\mathrm{~N}$ \\
\hline Unaffected & III-5 & $\mathrm{N}$ & $\mathrm{N}$ & $\mathrm{N}$ & $\mathrm{N}$ & $\mathrm{N}$ & $\mathrm{N}$ \\
\hline Affected & III-6 & Both & Both & $\mathrm{N}$ & $Y$ & $Y$ & $Y$ \\
\hline
\end{tabular}

TABLE 2 | Summary and bioinformatic analysis of the pathogenic variants identified in Families A-F.

\begin{tabular}{|c|c|c|c|c|c|c|c|c|c|c|}
\hline Family & Gene & $\begin{array}{c}\text { Reference } \\
\text { transcription }\end{array}$ & $\begin{array}{c}\text { Candidate } \\
\text { variants }\end{array}$ & $\begin{array}{l}\text { The types } \\
\text { of splice } \\
\text { site } \\
\text { mutations }\end{array}$ & HSF $^{\mathbf{a}}$ & NetGene2 $^{a}$ & NNSPLICE ${ }^{a}$ & MAF & $\begin{array}{c}\text { ACMG } \\
\text { classification }\end{array}$ & $\begin{array}{c}\text { Expression } \\
\text { in blood }\end{array}$ \\
\hline A & EYA4 & NM_172103 & c.580G > A & $\begin{array}{l}\text { II } \\
\text { (junctional) }\end{array}$ & $\begin{array}{l}\text { Disruption of the } \\
\text { original donor } \\
\text { splice site }(0.78 \\
>0.67)\end{array}$ & $\begin{array}{l}\text { Cannot be } \\
\text { predicted }\end{array}$ & $\begin{array}{l}\text { Cannot be } \\
\text { predicted }\end{array}$ & 0 & Uncertain & $\begin{array}{l}\text { extremely } \\
\text { low }\end{array}$ \\
\hline$B$ & EYA4 & NM_172103 & $\begin{array}{c}\text { c. } 1616+3 G \\
>A\end{array}$ & $\begin{array}{l}\text { ॥ } \\
\text { (junctional) }\end{array}$ & $\begin{array}{l}\text { Disruption of the } \\
\text { original donor } \\
\text { splice site }(0.10 \\
>0.06)\end{array}$ & $\begin{array}{l}\text { weak change of } \\
\text { the original } \\
\text { splice site }(0.47 \\
>0.41)\end{array}$ & $\begin{array}{l}\text { weak change of } \\
\text { the original splice } \\
\text { site }(1.00>0.99)\end{array}$ & 0 & Uncertain & $\begin{array}{l}\text { extremely } \\
\text { low }\end{array}$ \\
\hline C & DFNA5 & NM_004403 & $\begin{array}{c}\text { c.991- } \\
\text { 15_991- } \\
\text { 13del }\end{array}$ & $\begin{array}{l}\text { IV (deep } \\
\text { intronic) }\end{array}$ & $\begin{array}{l}\text { Creation of a } \\
\text { new acceptor } \\
\text { site }(0.52 \\
>0.88)\end{array}$ & $\begin{array}{l}\text { No change of the } \\
\text { original splice } \\
\text { site }(1.00>1.00)\end{array}$ & $\begin{array}{l}\text { No change of the } \\
\text { original splice site } \\
(0.93>0.94)\end{array}$ & 0 & Uncertain & $\begin{array}{l}\text { extremely } \\
\text { low }\end{array}$ \\
\hline$D$ & PAX3 & NM_181459 & $\begin{array}{c}\text { c.322- } \\
57 \_322-8 d e l\end{array}$ & $\begin{array}{l}\text { IV (deep } \\
\text { intronic) }\end{array}$ & $\begin{array}{l}\text { Creation of a } \\
\text { new acceptor } \\
\text { site }(0.30 \\
>0.84)\end{array}$ & $\begin{array}{l}\text { Disruption of the } \\
\text { original splice } \\
\text { site }(0.43>0)\end{array}$ & $\begin{array}{l}\text { Disruption of the } \\
\text { original splice site } \\
(0.52>0)\end{array}$ & 0 & Uncertain & $\begin{array}{l}\text { extremely } \\
\text { low }\end{array}$ \\
\hline$E$ & PTPRQ & NM_001145026 & $\begin{array}{c}\text { c. } 5426+1 G \\
>A\end{array}$ & I (canonical) & $\begin{array}{l}\text { Disruption of the } \\
\text { original splice } \\
\text { site }(0.89 \\
>0.63)\end{array}$ & $\begin{array}{l}\text { Disruption of the } \\
\text { original splice } \\
\text { site }(0.47>0)\end{array}$ & $\begin{array}{l}\text { Disruption of the } \\
\text { original splice site } \\
(0.99>0)\end{array}$ & 0 & $\begin{array}{c}\text { likely } \\
\text { pathogenic }\end{array}$ & $\begin{array}{l}\text { extremely } \\
\text { low }\end{array}$ \\
\hline$E$ & PTPRQ & NM_001145026 & $\begin{array}{c}\text { c. } 6087-3 \mathrm{~T} \\
>\mathrm{G}\end{array}$ & $\begin{array}{l}\text { ॥ } \\
\text { (junctional) }\end{array}$ & $\begin{array}{l}\text { Creation of a } \\
\text { new acceptor } \\
\text { site }(0.61 \\
>0.66)\end{array}$ & $\begin{array}{l}\text { Disruption of the } \\
\text { original splice } \\
\text { site }(0.26>0)\end{array}$ & $\begin{array}{l}\text { Disruption of the } \\
\text { original splice site } \\
(0.93>0)\end{array}$ & 0 & Uncertain & $\begin{array}{l}\text { extremely } \\
\text { low }\end{array}$ \\
\hline $\mathrm{F}$ & USH1G & NM_173477 & $\begin{array}{c}\text { c. } 164+5 G \\
>A\end{array}$ & $\begin{array}{l}\text { ॥ } \\
\text { (junctional) }\end{array}$ & $\begin{array}{l}\text { Disruption of the } \\
\text { original splice } \\
\text { site }(0.91 \\
>0.81)\end{array}$ & $\begin{array}{l}\text { Disruption of the } \\
\text { original splice } \\
\text { site }(1.00>0.71)\end{array}$ & $\begin{array}{l}\text { Disruption of the } \\
\text { original splice site } \\
(0.99>0)\end{array}$ & 0 & Uncertain & $\begin{array}{l}\text { extremely } \\
\text { low }\end{array}$ \\
\hline
\end{tabular}

aThe numbers in the parentheses indicate the confidence scores of a newly created or original splicing site before and after mutation, which range from 0 (strongly disruptive) to 1 (strongly supportive).

including c.580G > A in EYA4 for Family A, c. $1616+3 \mathrm{~A}>\mathrm{T}$ in EYA4 for Family B, c.991-15_991-13del in DFNA5 for Family $\mathrm{C}$, and c.322-57_322-8del in PAX3 for Family D. Cosegregation of the variants and the disease phenotype were confirmed in all participating family members (Figure 1). The audiograms and associating features (the vestibular disorder) in Family C and the WS3-associated phenotype in Family D are consistent with previously reported genotype-phenotype correlation for the corresponding genes (Somashekar et al., 2020). In the two simplex families, compound heterozygous variants c. $5426+1 \mathrm{G}>\mathrm{A}$ and c.6087-3T > G in PTPRQ were identified in the proband of Family $\mathrm{E}$ and homozygous variant c. $164+5 \mathrm{G}>\mathrm{A}$ in USH1G in Family F. No other candidate variants in known deafness genes have been identified. 


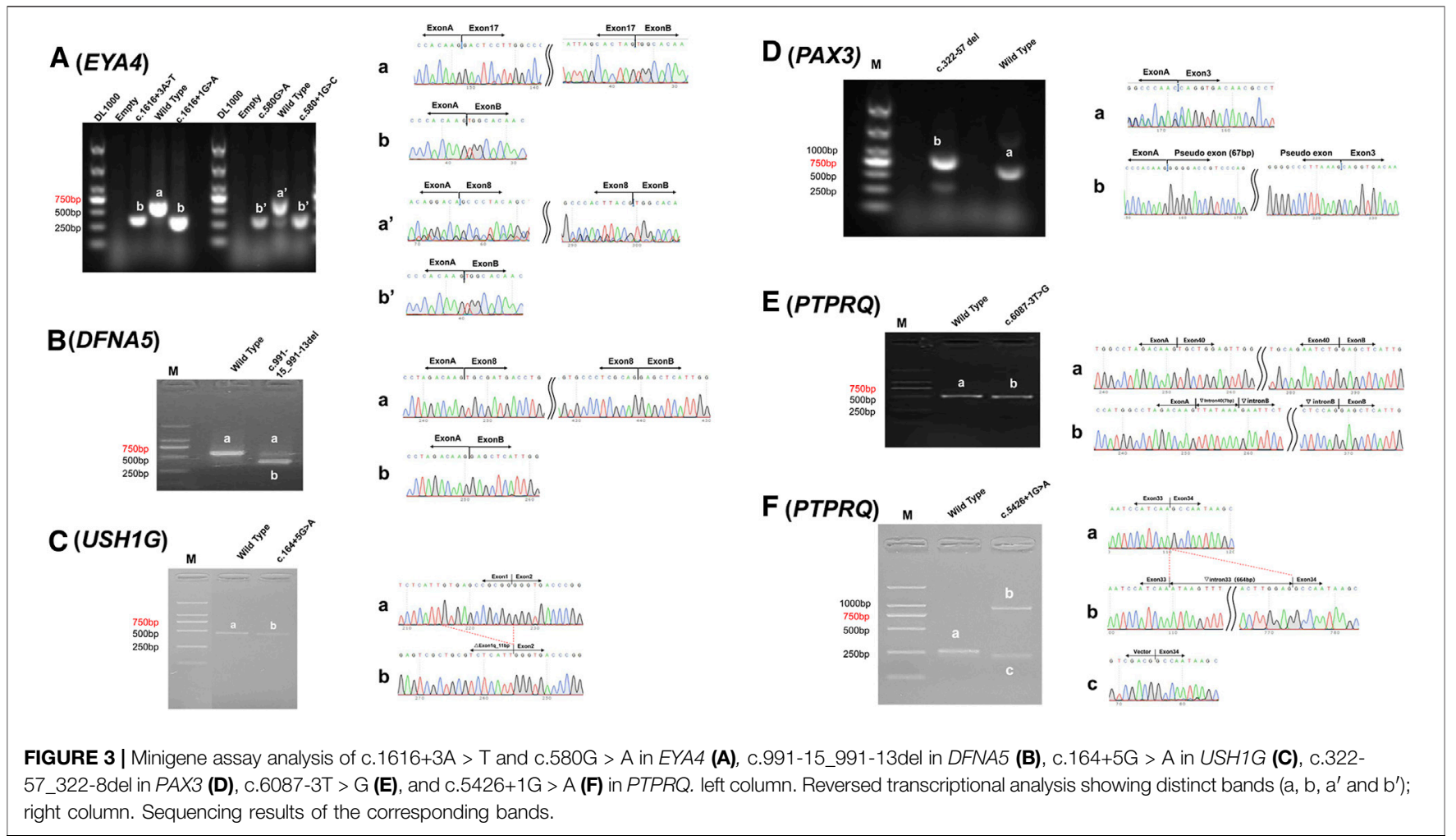

\section{Bioinformatic Analysis for the Pathogenicity of the Candidate Variants}

Results of the bioinformatic analysis were shown in Table 2. Because of the close vicinity of the EYA 4 c. $580 \mathrm{G}>$ A variant right next to the donor splice site, here we evaluated its potential negative effect on splicing instead of the presumed amino acid substitution (p.Asp194Asn). Beside the canonical c.5426+1G > A of $P T P R Q$ variant, the rest of the six variants identified in this study were all type II (c.580G $>$ A of EYA4, c.1616+3G $>$ A of EYA4, c.6087-3T > G of PTPRQ and c.164+5G > A of USH1G) or type IV (c.991-15_991-13del of DFNA5 and c.322-57_322-8del of $P A X 3)$ non-canonical splice site mutations. For the noncanonical splice site variants, HSF predicts that c.6087-3T > G of PTPRQ, c.991-15_991-13del of DFNA5 and c.322-57_322-8del of $P A X 3$ create new acceptor sites, while c.580G > A of EYA4, c. $1616+3 \mathrm{G}>\mathrm{A}$ of $E Y A 4$ and c.164+5G $>$ A of $U S H 1 G$ disrupt the original splice sites. NetGene2 and NNSPLICE predict that c.1616+3G > A of EYA4, c.322-57_322-8del of $P A X 3$, c.6087$3 \mathrm{~T}>\mathrm{G}$ of PTPRQ and c.164+5G > A of USH1G (weakly) disrupted the original splice sites. None of the variants were present in public databases ESP (http://evs.gs.washington.edu/ EVS/), GnomAD (https://gnomad.broadinstitute.org/) and the in-house Chinese Han population database of 1,000 individuals.

\section{Verification of Disrupted Splicing for the NCSS Variants}

For all pathogenic variants identified in this study, the associated genes have extremely low expression in peripheral blood
(Table 2), preventing in vivo analysis directly using samples from the patients. By in vitro minigene assay, the c.1616+3A > T and c.580G > A variants in EYA4 and the c.991-15_991-13del variant in DFNA5 generated a shorter spliced product missing the entire exon according to the sequencing results (Figures $\mathbf{3 A}, \mathbf{B}$ ). These three variants probably disrupt their original splicing site and lead to exon skipping. The rest four candidate variants generated spliced products with more complicated pattern, including exon shortening of $11 \mathrm{bp}$ from the $3^{\prime}$ end for c. $164+5 \mathrm{G}>\mathrm{A}$ in $U S H 1 G$ (Figure 3C), intron retention of $67 \mathrm{bp}$ from the $5^{\prime}$ end for c.322-57_322-8del in PAX3 (Figure 3D), exon skipping and partial intron retention for both c. $5426+1 \mathrm{G}>\mathrm{A}$ and c.6087-3T > G variants in PTPRQ (Figures 3E,F). These four variants probably disrupt the original splice sites and activate alternative ones in the exon or intron.

\section{DISCUSSION}

Targeted or whole-exome NGS has been increasingly employed for mutation screening of heterogeneous diseases such as deafness. The routine sequencing data analysis usually focus on non-synonymous variants in exon and CSS variant only, while the potential NCSS variants are often ignored. Though the exact prevalence of the NCSS pathogenic variants is not clear for hereditary hearing loss, several previous reports and our current study has suggested that it very likely be notable, especially in familial cases with no pathogenic variants identified in known deafness genes (Booth et al., 2018). In this paper, we propose a workflow for detection and verification of 
NCSS mutations by NGS (Supplementary Figure S1). The modified filtering criteria evaluate all variants, intronic or exonic, synonymous or non-synonymous, for potential negative effect on splicing, which will be followed by bioinformatic analysis and functional verification by either reversed transcriptional PCR or minigene analysis. Other helpful criteria include intrafamilial phenotypic cosegregation, consistency with previously reported genespecific genotype-phenotype correlation, and identification of pathogenic mutations in both alleles of the recessive causative genes.

In this study, multiplex, autosomal dominant families A-D have pedigrees large enough for effective intrafamilial phenotypic co-segregation analysis. The compound heterozygous (PTPRQ) or homozygous (USH1G) mutations in Family $\mathrm{E}$ and $\mathrm{F}$ are in agreement with the presumable recessive inheritance as reported by numerous previously studies (Sang et al., 2015; Wu et al., 2018; D'Esposito et al., 2021). Affected members within each family have audiograms that show similar hearing phenotype and are consistent with previous reports for genotype-phenotype correlation of EYA4, DFNA5, PAX3, PTPRQ and USH1G. Family D has additional features perfectly matched to WS3, for which PAX3 is the main causative gene. With these supporting evidences, we were able to narrow down a list of candidate NCSS variants including several that were originally missed by routine NGS filtering process.

In this paper, we predicted the pathogenicity of the six NCSS variants by three computational prediction software HSF, NNSPLICE and NetGene2 (Table 2). Though all six variants were predicted to affect the RNA splicing by at least one computational tool, it remains to be functionally verified as prediction tools alone frequently generate false positive or false negative results. In this aspect, the simplest and most effective approach is targeted sequences reversed transcriptional PCR in tissues of the lesion (Valero et al., 2019). This approach, however, rarely works for deafnesscausative genes, as the cochlear tissue is difficult to obtain and none of five genes involved in this study are expressed in the peripheral blood. In this case, minigene analysis provides an in vitro assay for testing RNA splicing effect of NCSS variants, which can be used to confirm whether potential pathogenic variants affect splicing efficiency or activate variable hidden splicing sites (Booth et al., 2018). It also remains to be verified by other hearing-impaired patients carrying the same NCSS variants, which will provide further genetic evidence in support for its pathogenicity.

Based on the minigene analysis results, we concluded that splice site variants at the classical (type I) and junction (type II) region often directly destroy the original acceptor or donor splice site, leading to exon skipping, such as the c.1616+3A $>\mathrm{T}$ and c.580G > A variants in EYA4 (Figure 3A). Sometimes NCSS variants can weaken the recognition of the donor or acceptor splicing site and activate a cryptic splicing site in the intron, resulting in exon skipping and/or partial intron retention, such as the c.5426+1G > A and c.6087-3T > G variants in PTPRQ (Figure 3F), or activate a cryptic splicing site in the exon, resulting in shortening of the exon, such as the c. 164+5G > A variant in USH1G (Figure 3C). Mutations in the deep intron region (type IV) can destroy the branch point with polypyrimidine tract, resulting in exon skipping, such as c.99115_991-13del in DFNA5 (Figure 3B), or activate a cryptic splicing site in the deep intron, resulting in partial intron retention, such as the c.322-57_322-8del variant in $P A X 3$ (Figure 3D).

The c.580G > A variant in EYA4 is of particular interest, as under most circumstance it will be interpreted as a p. Asp194Asn missense mutation at a very conservative amino acid position. Though most of the reported EYA4 mutations are truncating mutations leading to haploinsufficiency (Zhang et al., 2004), at least four EYA4 missense mutations have been reported to lead to non-syndromic deafness DFNA10(Tan et al., 2014; Liu et al., 2015; Sun et al., 2015; Cesca et al., 2018). However, our results showed that the c.580C > G variant disrupts the pre-mRNA splicing of EYA4, resulting in exon 8 (143 bases) skipping and presumably early termination of the protein translation. In light of this discovery, we designed minigene experiment to analyze the other four previously reported EYA4 missense mutations c.511G > C, c.978C > G, c.1301T > A and c.1643C > G, which all tested negative for any splicing abnormality (Supplementary Figure S2). Nevertheless, it is worth noting that exonic synonymous variants or benign amino acid substitutions may be hidden NCSS variants such as the c.580C > G variant in EYA4, which may deserve reevaluation and in-depth study.

In conclusion, we identified and functionally verified 6 NCSS variants in EYA4, PAX3, PTPRQ, and USH1G in Chinese Han families with sensorineural deafness. The NCSS variants may be an important cause of genetic hearing loss that demands closer attention in genetic diagnosis.

\section{DATA AVAILABILITY STATEMENT}

The original contributions presented in the study are publicly available. This data can be found here: PRJNA779459.

\section{ETHICS STATEMENT}

The studies involving human participants were reviewed and approved by the ethics committee of Ninth People's Hospital, Shanghai Jiaotong University School of Medicine. Written informed consent to participate in this study was provided by the participants' legal guardian/next of kin.

\section{AUTHOR CONTRIBUTIONS}

PC collected and analyzed the clinical and experiment data and wrote the paper, LW and YC helped collected the clinical data and genetic analysis, HW was responsible for the study conception. TY was responsible for data quality control and wrote the manuscript. PC, LW, and YC contributed equally to this work. 


\section{FUNDING}

This work is supported by grants from National Science Foundation of China (81970894 to TY, 82000989 to PC), National Key R\&D Program of China (2017YFC1001804 to HW), Shanghai Municipal Science and Technology Commission (14DZ2260300 to HW), Shanghai Municipal Education Commission-Gaofeng Clinical Medicine Grant (20152519 to TY) and Shanghai Sailing Program (20YF1428800 to PC).

\section{ACKNOWLEDGMENTS}

We appreciated the grants from National Natural Science Foundation of China (81970894 to TY, 82000989 to PC),

\section{REFERENCES}

Anna, A., and Monika, G. (2018). Splicing Mutations in Human Genetic Disorders: Examples, Detection, and Confirmation. J. Appl. Genet. 59, 253-268. doi:10.1007/s13353-018-0444-7

Booth, K. T., Azaiez, H., Kahrizi, K., Wang, D., Zhang, Y., Frees, K., et al. (2018). Exonic Mutations and Exon Skipping: Lessons Learned from DFNA5. Hum. Mutat. 39, 433-440. doi:10.1002/humu.23384

Cartegni, L., Chew, S. L., and Krainer, A. R. (2002). Listening to Silence and Understanding Nonsense: Exonic Mutations that Affect Splicing. Nat. Rev. Genet. 3, 285-298. doi:10.1038/nrg775

Cesca, F., Bettella, E., Polli, R., Cama, E., Scimemi, P., Santarelli, R., et al. (2018). A Novel Mutation of the EYA4 Gene Associated with post-lingual Hearing Loss in a Proband Is Co-segregating with a Novel PAX3 Mutation in Two Congenitally Deaf Family Members. Int. J. Pediatr. Otorhinolaryngol. 104, 88-93. doi:10.1016/j.ijporl.2017.10.042

Chen, P., He, L., Pang, X., Wang, X., Yang, T., and Wu, H. (2016). NLRP3 Is Expressed in the Spiral Ganglion Neurons and Associated with Both Syndromic and Nonsyndromic Sensorineural Deafness. Neural Plasticity 2016, 1-6. doi:10.1155/2016/3018132

D’Esposito, F., Randazzo, V., Cennamo, G., Centore, N., Maltese, P. E., Malesci, R., et al. (2021). Novel USH1G Homozygous Variant Underlying USH2-like Phenotype of Usher Syndrome. Eur. J. Ophthalmol. 31, NP18-NP22. doi:10.1177/1120672119879392

Glisovic, T., Bachorik, J. L., Yong, J., and Dreyfuss, G. (2008). RNA-binding Proteins and post-transcriptional Gene Regulation. FEBS Lett. 582, 1977-1986. doi:10.1016/j.febslet.2008.03.004

Hildebrand, M. S., Coman, D., Yang, T., Gardner, R. J. M., Rose, E., Smith, R. J. H., et al. (2007). A Novel Splice Site Mutation inEYA4 Causes DFNA10 Hearing Loss. Am. J. Med. Genet. 143A, 1599-1604. doi:10.1002/ ajmg.a.31860

Kremer, H. (2019). Hereditary Hearing Loss; about the Known and the Unknown. Hearing Res. 376, 58-68. doi:10.1016/j.heares.2019.01.003

Liu, F., Hu, J., Xia, W., Hao, L., Ma, J., Ma, D., et al. (2015). Exome Sequencing Identifies a Mutation in EYA4 as a Novel Cause of Autosomal Dominant Nonsyndromic Hearing Loss. PLoS ONE 10, e0126602. doi:10.1371/ journal.pone.0126602

Sang, Q., Mei, H., Kuermanhan, A., Feng, R., Guo, L., Qu, R., et al. (2015). Identification of a Novel Compound Heterozygous Mutation in PTPRQ in a DFNB84 Family with Prelingual Sensorineural Hearing Impairment. Mol. Genet. Genomics 290, 1135-1139. doi:10.1007/s00438-014-0979-1

Shearer, A. E., Eppsteiner, R. W., Booth, K. T., Ephraim, S. S., Gurrola, J., Simpson, A., et al. (2014). Utilizing Ethnic-specific Differences in Minor Allele Frequency to Recategorize Reported Pathogenic Deafness Variants. Am. J. Hum. Genet. 95, 445-453. doi:10.1016/j.ajhg.2014.09.001

Sheffield, A. M., and Smith, R. J. H. (2019). The Epidemiology of Deafness. Cold Spring Harb Perspect. Med. 9, a033258. doi:10.1101/cshperspect.a033258
National Key R\&D Program of China (2017YFC1001804 to HW), Shanghai Municipal Science and Technology Commission (14DZ2260300 to HW), Shanghai Municipal Education Commission-Gaofeng Clinical Medicine Grant (20152519 to TY) and Shanghai Sailing Program (20YF1428800 to PC).

\section{SUPPLEMENTARY MATERIAL}

The Supplementary Material for this article can be found online at: https://www.frontiersin.org/articles/10.3389/fgene.2021.773922/ full\#supplementary-material

Somashekar, P. H., Upadhyai, P., Narayanan, D. L., Kamath, N., Bajaj, S., Girisha, K. M., et al. (2020). Phenotypic Diversity and Genetic Complexity of PAX3 -related Waardenburg Syndrome. Am. J. Med. Genet. 182, 2951-2958. doi:10.1002/ajmg.a.61893

Sun, Y., Zhang, Z., Cheng, J., Lu, Y., Yang, C.-L., Luo, Y.-Y., et al. (2015). A Novel Mutation of EYA4 in a Large Chinese Family with Autosomal Dominant Middle-Frequency Sensorineural Hearing Loss by Targeted Exome Sequencing. J. Hum. Genet. 60, 299-304. doi:10.1038/jhg.2015.19

Tan, M., Shen, X., Yao, J., Wei, Q., Lu, Y., Cao, X., et al. (2014). Identification of I411K, a Novel Missense EYA4 Mutation Causing Autosomal Dominant Non-syndromic Hearing Loss. Int. J. Mol. Med. 34, 1467-1472. doi:10.3892/ijmm.2014.1939

Valero, R., de Castro-Miró, M., Jiménez-Ochoa, S., Rodríguez-Ezcurra, J. J., Marfany, G., and Gonzàlez-Duarte, R. (2019). Aberrant Splicing Events Associated to $\mathrm{CDH} 23$ Noncanonical Splice Site Mutations in a Proband with Atypical Usher Syndrome 1. Genes 10, 732. doi:10.3390/ genes10100732

Vaz-Drago, R., Custódio, N., and Carmo-Fonseca, M. (2017). Deep Intronic Mutations and Human Disease. Hum. Genet. 136, 1093-1111. doi:10.1007/ s00439-017-1809-4

Wang, J., Zhang, J., Li, K., Zhao, W., and Cui, Q. (2012). SpliceDisease Database: Linking RNA Splicing and Disease. Nucleic Acids Res. 40, D1055-D1059. doi:10.1093/nar/gkr1171

Wu, X., Wang, S., Chen, S., Wen, Y.-y., Liu, B., Xie, W., et al. (2018). Autosomal Recessive Congenital Sensorineural Hearing Loss Due to a Novel Compound Heterozygous PTPRQ Mutation in a Chinese Family. Neural Plasticity 2018, 1-6. doi:10.1155/2018/9425725

Zhang, Y., Knosp, B. M., Maconochie, M., Friedman, R. A., and Smith, R. J. H. (2004). A Comparative Study of Eyal and Eya4 Protein Function and its Implication in Branchio-Oto-Renal Syndrome and DFNA10. Jaro 5, 295-304. doi:10.1007/s10162-004-4044-3

Conflict of Interest: The authors declare that the research was conducted in the absence of any commercial or financial relationships that could be construed as a potential conflict of interest.

Publisher's Note: All claims expressed in this article are solely those of the authors and do not necessarily represent those of their affiliated organizations, or those of the publisher, the editors and the reviewers. Any product that may be evaluated in this article, or claim that may be made by its manufacturer, is not guaranteed or endorsed by the publisher.

Copyright (C) 2021 Chen, Wang, Chai, Wu and Yang. This is an open-access article distributed under the terms of the Creative Commons Attribution License (CC BY). The use, distribution or reproduction in other forums is permitted, provided the original author(s) and the copyright owner(s) are credited and that the original publication in this journal is cited, in accordance with accepted academic practice. No use, distribution or reproduction is permitted which does not comply with these terms. 